\title{
Editorial: Advanced Industrial Networks with loT and Big Data
}

\author{
Yan Zhang ${ }^{1} \cdot$ Mithun Mukherjee $^{2} \cdot$ Celimuge $\mathrm{Wu}^{3} \cdot$ Ming-Tuo Zhou ${ }^{4}$ \\ Published online: 22 April 2019 \\ (C) Springer Science+Business Media, LLC, part of Springer Nature 2019
}

\section{Editorial:}

Internet of Thing (IoT) has the potential to deliver exciting things across many sectors, from industry to social media and home. These networks of things are designed to measure realworld events and expected to control more than two billion connected devices to the internet by 2020 . Due to the advantage of low-cost, easy to deploy, energy efficiency and mobility compared to the traditionally applied field bus, industrial wireless sensor network has become a promising approach for manufactures as well as plant designers. With the paradigm of IoT, Industrial Wireless Sensor Networks (IWSNs) are evolving to the global interconnection between management and factory products in the large-scale industry. It serves as a link between data collected from heterogeneous sources on site and business backend. Recently, IWSN integrated with IoT and Big Data is an attractive choice for industrial processes. The large-scale industry consists of dense wireless devices such as RFID tags for machine identification, sensors used for large-scale rotational equipment monitoring and fault diagnosis and many more. With the data acquisition across heterogeneous sources and intelligent processing of gathered data, one important aspect is to predict any dangerous situation like leakage of toxic gas in large scale pipeline. Furthermore, the layout design procedure in the industry to ensure connectivity and information flow is also a part of IoT paradigm. Thus, the primary concerns of IWSN integrated with IoT and Big Data are capability, reliability, and cost. This special issue focuses on exploring recent emerging technologies and research developments on industrial

Yan Zhang

yanzhang@ifi.uio.no

1 Department of Informatics, University of Oslo, Oslo, Norway

2 University Institute of Technology, Burdwan University, Bardhaman, India

3 Beijing Institute of Technology, Beijing, China

4 Shanghai Institute of Microsystem and Information Technology (SIMIT), Chinese Academy of Sciences (CAS), Shanghai, China networks and intelligent systems to advance the step towards the smarter plants integrated with IoT and Big Data.

This special issue selects six high-quality papers. The first article titled "Optimal and Elastic Energy Trading for Green Microgrids: A two-layer Game Approach" authored by Zhou et al. discusses the energy trading mechanism for microgrids. The article employs a two-layer game approach to achieve optimal and elastic energy trading for microgrids and significantly improved the utilization of microgrids' green energy. The proposed algorithms can reach the equilibriums where the players achieve optimal utilities. Finally, the existence and uniqueness of the equilibriums in the energy trading mechanism for microgrids are discussed.

Cloud-Radio Access Networks (C-RANs) that becomes an attractive solution to meet the enormous demand for diverse communications decouples the BaseBand processing Unit (BBU) from the Remote Radio Head (RRH). In the article "When Green Energy Meets Cloud Radio Access Network: Joint Optimization Towards Brown Energy Minimization", Guo et al. consider the network energy consumption and investigated a joint RRH-BBU association and energy sharing problem towards brown energy usage minimization in green energy powered C-RAN. A two-phase heuristic polynomial-time algorithm is proposed to minimize the computation complexity of solving mixed integer linear programming (MILP).

The third article titled "Diffusion Distance-Based Predictive Tracking for Continuous Objects in Industrial Wireless Sensor Networks" by Liu et al. proposes a diffusion distance-based predictive tracking algorithm is proposed for Industrial Wireless Sensor Networks (IWSNs) to track the boundary of a continuous object after the occurrence of a leak. To achieve high energy-efficiency, a sleep-wake cycle is introduced to select a subset of nodes in the process of tracking, while the rest of the nodes stay idle until an object approaches. Finally, a clusterbased competitive mechanism is proposed for reporting the location of boundary nodes.

In the next article with the title "A Simplified Interference Model for Outdoor Millimeter-wave Networks", Jiang et al. consider the Millimeter-wave (mmWave) communication that becomes an emerging technology for wireless networks to 
support the Industry 4.0 implementation. They investigated the accuracy of an interference model for outdoor mmWave networks that assumes impenetrable obstacles and neglects the sidelobes. They quantify the error of regarding statistical distribution of the signal-to-noise-plus-interference ratio for outdoor mmWave networks under different antenna array settings.

The fifth article titled "Data Driven Cyber-Physical System for Landslide Detection" introduces a data-driven cyber-physical system is introduced to detect landslides, one of the most dangerous natural disasters. This proposed system is composed of Wi-Sun acceleration sensors to detect the acceleration of the nearby environment in a 3D domain. Moreover, low computational complexity-based landslide detection mechanism is proposed. The proposed scheme aims to save the energy consumption without any significant degradation in the detection performance. Furthermore, results from the field test verify the system's effectiveness.

The last article titled "Contract-theoretic Approach for Delay Constrained Offloading in Vehicular Edge Computing Networks" by Zhang et al. proposes a new Vehicular Edge Computing (VEC) framework to model the computation offloading process of the mobile vehicles running on a bidirectional road. They adopted a contract theoretic approach to design optimal offloading strategies for the VEC service provider, to maximize the revenue of the provider while enhancing the utilities of the vehicles. To further improve the utilization of the computing resources of the VEC servers, the authors incorporate task priority distinction as well as an additional resource providing into the design of the offloading scheme.

Acknowledgements The guest editors are thankful to our reviewers for their effort in reviewing the manuscripts. We also thank the Edit-in-Chief, Dr. Imrich Chlamtac for his supportive guidance during the entire process.

Publisher's Note Springer Nature remains neutral with regard to jurisdictional claims in published maps and institutional affiliations.

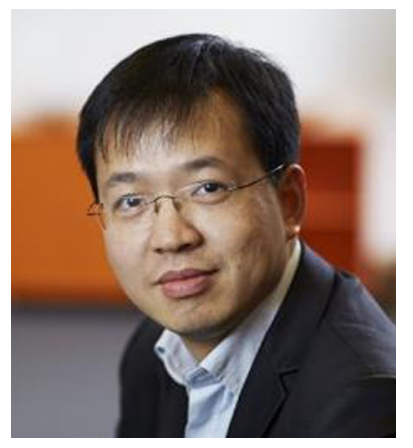

Yan Zhang is Full Professor at the Department of Informatics, University of Oslo, Norway. He received a $\mathrm{PhD}$ degree in School of Electrical \& Electronics Engineering, Nanyang Technological University, Singapore. He is an Associate Technical Editor of IEEE Communications Magazine, an Editor of IEEE Network Magazine, an Editor of IEEE Transactions on Green Communications and Networking, an Editor of IEEE Communications Surveys \& Tutorials, an Editor of IEEE Internet of Things Journal, an Editor of IEEE Vehicular Technology Magazine, and an Associate Editor of IEEE Access. He serves as chair positions in a number of conferences, including IEEE GLOBECOM 2017, IEEE VTCSpring 2017, IEEE PIMRC 2016, IEEE CloudCom 2016, IEEE ICCC 2016, IEEE CCNC 2016, IEEE SmartGridComm 2015, and IEEE CloudCom 2015. He serves as TPC member for numerous international conference including IEEE INFOCOM, IEEE ICC, IEEE GLOBECOM, and IEEE WCNC. His current research interests include: next-generation wireless networks leading to $5 \mathrm{G}$, green and secure cyber-physical systems (e.g., smart grid, healthcare, and transport). He is IEEE VTS (Vehicular Technology Society) Distinguished Lecturer. He is also a senior member of IEEE, IEEE ComSoc, IEEE CS, IEEE PES, and IEEE VT society.

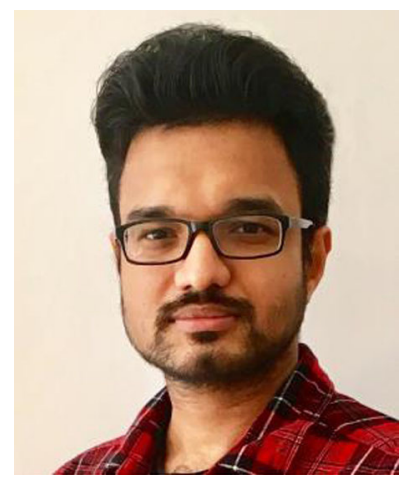

Mithun Mukherjee received his B.E. degree in electronics and communication engineering from the University Institute of Technology, Burdwan University, India, in 2007, his M.E. degree in information and communication engineering from the Indian Institute of Science and Technology, Shibpur, in 2009, and his Ph.D. degree in electrical engineering from the Indian Institute of Technology Patna, in 2015. Currently, he is a specially assigned researcher in the Guangdong Provincial Key Lab of Petrochemical Equipment Fault Diagnosis, Guangdong University of Petrochemical Technology. He was a recipient of the EAI WICON, the 2017 IEEE SigTelCom Best Paper Award, and the 2018 IEEE Systems Journal Best Paper Award. He was a Guest Editor of the IEEE TRANSACTIONS ON INDUSTRIAL INFORMATICS, ACM/Springer Mobile Networks \& Applications, and Sensors, and an Associate Editor of IEEE Access. His research interests include wireless communications, energy harvesting, and fog computing.

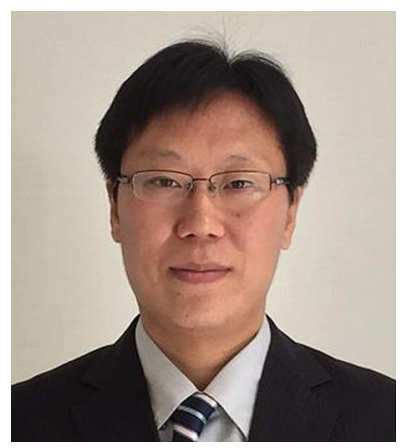

Celimuge $\mathrm{Wu}$ received the M.E. degree from Beijing Institute of Technology, Beijing, China, in 2006, and the $\mathrm{PhD}$ degree from the University of ElectroCommunications, Tokyo, Japan, in 2010. Since 2010, he has been an assistant professor at the Graduate School of Information Systems, the University of Electro-Communications. He has been serving as an expert member of IEICE Technical Committee on Communication Quality since 2013, and as a technical program committee member for many prestigious conferences including IEEE GLOBECOM, IEEE ICC, IEEE VTC, IEEE PIMRC etc. He received IPSJ Digital Courier Funai Young Researcher Encouragement Award in 2011. His current research interests include wireless networks, networking architectures and protocols. 


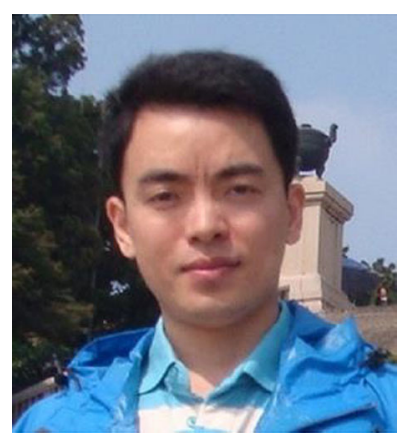

Ming-tuo Zhou received his $\mathrm{PhD}$ degree in 2003. He joined Shanghai Institute of Microsystem and Information Technology (SIMIT), Chinese Academy of Sciences (CAS) in September 2016, and now is a professor and the head of the Computing \& Communication Research Group. He is also affiliated with the Shanghai Institute of Fog Computing Technology (SHIFT), a joint lab of ShanghaiTech University and SIMIT. He was a senior research scientist at the Smart Wireless Laboratory of the (Japan) National Institute of Information and Communications Technology (NICT) Singapore
Representative Office during July 2004 to August 2016. He is the chair of Testbed Working Group of OpenFog Consortium since September 2017 and the Secretary of IEEE 1934 working group since December 2018. His current research interests include fog computing and communications, wireless sensor networks and Internet of things. He is an IEEE SA member and an IEEE Senior Member. 